\title{
Potent Corticosteroids Inhibit Lipogenesis in Sebaceous Glands
}

\author{
WenChieh Chen ${ }^{a}$ Chen-Yu Liao ${ }^{a}$ Chiu-Ling Hung ${ }^{a}$ Tzu-Kai Lin ${ }^{b}$ \\ Hamm-Ming Sheu ${ }^{c}$ Christos C. Zouboulis ${ }^{d}$ \\ aDepartment of Dermatology, Chang Gung Memorial Hospital, Kaohsiung, Chang Gung University, Taoyuon, \\ ${ }^{b}$ Department of Dermatology, Tzu-Chi Buddhist General Hospital, Ta-Lin, and 'Department of Dermatology, \\ College of Medicine, National Cheng Kung University, Tainan, Taiwan; ${ }^{\mathrm{d}}$ Departments of Dermatology and \\ Immunology, Dessau Medical Center, Dessau, Germany
}

\section{Key Words}

Glucocorticoids · Lipogenesis · Sebum • Steroid acne •

Triglycerides

Steroid acne is the most common form of drug-induced acne. It may appear as a side effect of short-term or long-term, topical or systemic administration of corticosteroids for medical (iatrogenic) or illicit (doping) purposes. Steroids of higher potency and at higher doses are more capable of inducing acne formation [1]. Histopathological studies in the 1970s showed that steroid acne was initiated by inflammation of the mid-infundibulum of the sebaceous follicle, followed by formation of closed comedones, accompanied with atrophy of sebaceous glands [1]. On the other hand, hydrocortisone was known to be an essential supplement for the primary culture of sebocytes [2]. How steroids cause an atrophic sebaceous gland remains incompletely defined at the molecular level.

Lipid synthesis and accumulation of lipid droplets represent a major step in sebocyte differentiation, leading eventually to holocrine secretion of lipids as sebum. Two families of transcription factors, CCAAT/enhancer binding proteins and peroxisome proliferator-activated receptors, have been demonstrated to play a crucial role in the differentiation of adipocytes as well as sebocytes [3]. The sebum composition in mice and in humans differs. In hairless rhino mice, it comprises mostly wax esters (70\%), free sterols (13\%), sterol esters (10\%) and triglycerides (6\%) [4]. In humans, however, the three most abundant lipids in sebum are triglycerides (56\%), followed by wax esters (26\%) and squalene (15\%) [5].

In scanning electron microscopy, significant shrinkage by an average $36 \%$ was identified in the size of the bior trilobulated sebaceous glands after topical application of $0.064 \%$ betamethasone dipropionate ointment onto mouse skin for 6 weeks. Sebum analysis in the treatment group by high-performance thin-layer chromatography showed reduction of the amount of triglycerides, wax diester and sterol ester by 39, 64 and 64\%, respectively. In Nile red staining of the cultured immortalized SZ95 sebocytes, a decrease in lipid particles and cell size was evident after treatment with $10^{-5} \mathrm{M}$ betamethasone dipropionate or clobetasol propionate for 7 days. The differentiation-opposing effect of clobetasol propionate in particular could be partially reversed by addition of $10^{-7} \mathrm{M}$ linoleic acid. In flow cytometry, quantification of the lipid amount using the Nile red stain, linoleic acid at $10^{-4} \mathrm{M}$ could completely antagonize the suppressive effect of corticosteroids and boost lipid production in sebocytes. High-performance thin-layer chromatography analysis of the sebum composition in SZ95 sebocytes

\section{KARGER}

Fax +4161306 1234

E-Mail karger@karger.ch

www.karger.com (c) 2006 S. Karger AG, Basel

$1018-8665 / 06 / 2133-0264 \$ 23.50 / 0$

Accessible online at:

www.karger.com/drm
WenChieh Chen, MD

Department of Dermatology, Chang Gung Memorial Hospital

Kaohsiung, Ta-Pei Road 123, Niao-Sung

83301 Kaohsiung (Taiwan)

Tel. +886 7731 7123, ext. 2299, Fax +886 7733 7612, E-Mail wchen@cgmh.org.tw 
treated for 7 days demonstrated that triglyceride was the major lipid class that was attenuated by corticosteroids. In a Western blot study, the expression of peroxisome proliferator-activated receptors $\gamma_{1}$ and $\gamma_{2}$ was found to diminish considerably in sebocytes treated with betamethasone dipropionate. A 7-day treatment with various corticosteroids did not influence the proliferation of the cultured SZ95 sebocytes and their production of testosterone.

Our study seems to suggest that the exogenous synthetic corticosteroids exert their major antagonizing effect on sebocyte differentiation and sebum production. Conventionally the sebaceous gland is considered to be an atavistic structure with little or no biological function [5]. The role of sebaceous lipids is unlikely to be significant but to fuel acne flame [1]. However, clinical use of oral isotretinoin in the treatment of severe acne inducing extensive atrophy of sebaceous glands is always accompanied with skin dryness. More recent studies showed that knockout mice (DGAT-/- mice) with inactivated diacylglycerol acyltransferase, the main regulating enzyme for triglyceride synthesis, developed atrophic sebaceous glands, impaired water repulsion and defective thermoregulation after water immersion [6]. Glycerol derived from triglyceride turnover in sebaceous glands was found to be able to serve as a major contributor to stratum corneum hydration in asebia mice as well as in humans [7, 8]. These findings suggest a constitutive role of sebum in maintaining skin barrier homeostasis. On the other hand, it has been demonstrated that short-term and long-lasting use of topical corticosteroids could cause skin atrophy with fewer layers of stratum corneum, increase transepidermal water loss, and compromise both permeability and integrity of the stratum corneum by inhibition of epidermal lipid synthesis with diminishment of lipid content [9-11]. Therefore, the impairment of sebum synthesis precipitated by potent topical corticosteroids, as shown in the current study, may further aggravate and augment skin dryness caused by defective epidermal lipids.

Asteatosis reclaims its stand in contributing to xerosis of the skin.

\section{References}

1 Plewig G, Kligman AM: Acne and Rosacea, ed 3. Berlin, Springer, 2000.

2 Zouboulis CC, Xia L, Akamatsu H, Seltmann H, Fritsch M, Hornemann S, Ruhl R, Chen W, Nau H, Orfanos CE: The human sebocyte culture model provides new insights into development and management of seborrhoea and acne. Dermatology 1998;196:2131.

3 Chen W, Yang C-C, Sheu H-M, Seltmann H, Zouboulis CC: Expression of PPAR and $\mathrm{c} /$ EBP transcription factors in cultured human sebocytes. J Invest Dermatol 2003;121:441447.

4 Wilkinson DI, Karasek MA: Skin lipids of a normal and mutant (asebic) mouse strain. J Invest Dermatol 1966;47:449-455.

5 Cunliffe WJ, Gollnick HPM: Acne: Diagnosis and Management. London, Dunitz, 2001.
6 Chen HC, Smith SJ, Tow B, Elias PM, Farese RV Jr: Leptin modulates the effects of acylCoA:diacylglycerol acyltransferase deficiency on murine fur and sebaceous glands. J Clin Invest 2002;109:175-181.

7 Fluhr JW, Mao-Qiang M, Brown BE, Wertz PW, Crumrine D, Sundberg JP, Feingold KR, Elias PM: Glycerol regulates stratum corneum hydration in sebaceous gland deficient (asebia) mice. J Invest Dermatol 2003;120: 728-737.

8 Choi EH, Man MQ, Wang F, Zhang X, Brown BE, Feingold KR, Elias PM: Is endogenous glycerol a determinant of stratum corneum hydration in humans? J Invest Dermatol 2005; 125:288-293.

9 Sheu HM, Lee JY, Chai CY, Kuo KW: Depletion of stratum corneum intercellular lipid lamellae and barrier function abnormalities after long-term topical corticosteroids. Br J Dermatol 1997;136:884-890.
10 Sheu HM, Lee JY, Kuo KW, Tsai JC: Permeability barrier abnormality of hairless mouse epidermis after topical corticosteroid: characterization of stratum corneum lipids by ruthenium tetroxide staining and high-performance thin-layer chromatography. J Dermatol 1998;25:281-289.

11 Kao JS, Fluhr JW, Man MQ, Fowler AJ, Hachem JP, Crumrine D, Ahn SK, Brown BE, Elias PM, Feingold KR: Short-term glucocorticoid treatment compromises both permeability barrier homeostasis and stratum corneum integrity: inhibition of epidermal lipid synthesis accounts for functional abnormalities. J Invest Dermatol 2003;120: 456-464. 